\title{
Questes
}

\section{L'autorité d'une déesse antique : réflexions sur la figure de Vénus dans Le Livre du Cuer d'Amours espris}

Sophie Poitral

\section{OpenEdition}

Journals

Édition électronique

URL : http://journals.openedition.org/questes/1572

DOI : 10.4000/questes. 1572

ISSN : 2109-9472

Éditeur

Les Amis de Questes

Édition imprimée

Date de publication : 15 janvier 2006

Pagination : $39-48$

ISSN : 2102-7188

\section{Référence électronique}

Sophie Poitral, «L'autorité d'une déesse antique : réflexions sur la figure de Vénus dans Le Livre du Cuer d'Amours espris », Questes [En ligne], 8 | 2006, mis en ligne le 01 janvier 2014, consulté le 03 mai 2019. URL : http://journals.openedition.org/questes/1572 ; DOI : 10.4000/questes.1572

Ce document a été généré automatiquement le 3 mai 2019.

(C) Association des amis de «Questes » 


\title{
L'autorité d'une déesse antique : réflexions sur la figure de Vénus dans Le Livre du Cuer d'Amours espris
}

\author{
Sophie Poitral
}

"Aeneadum genetrix, hominum divomque
voluptas,
alma Venus, cæli subter labentia signa
quæ mare navigerum, quæ terras frugiferentis
concelebras, per te quoniam genus omne
animantum
concipitur visitque exortum lumina solis. »

Dans son Livre du Cuer d'Amours espris, René d'Anjou nous confie un songe qu'il a fait. Couché dans son lit, il lui semble qu'Amour, accompagné de son serviteur Désir, lui retire le cœur de la poitrine. À la suite de cet acte extraordinaire, l'organe devient un chevalier nommé Cœur qui part à travers un pays inconnu à la recherche de sa dame, Douce Merci. Après une série de péripéties, le chevalier et ses compagnons débarquent sur l'île d'Amour et pénètrent dans un château merveilleux, où vit Amour en compagnie de Vénus.

2 «Hault et puissant dieu terrien $»^{2}$, le « dieu d'Amours » jouit d'une autorité divine, tandis que Vénus, qui régnait jadis sur le mont Olympe parmi les plus grands dieux, est désignée sous l'appellation «dame Venus». Cette constatation sur les modalités de nomination d'Amour et de Vénus nous conduit à nous interroger sur l'autorité de cette dernière figure. L'objectif de notre étude sera de montrer l'importance du caractère mythologique dans la mise en place de l'autorité de Vénus dans l'imaginaire médiéval du duc d'Anjou. Dans cette perspective, nous aborderons d'abord brièvement son histoire, puis son implication dans la cour d'Amour, avant de nous attacher aux mystérieuses tapisseries qui ornent les murs de sa chambre. 


\section{Le poids de l'héritage païen}

3 Alors que dame Vénus s'apprête à regagner sa chambre, l'Acteur fournit le commentaire suivant: «(...) tant vous di je bien qu'elle estoit belle et habillee comme une deesse $»^{3}$. Cette comparaison n'est pas anodine, car elle est une référence directe aux origines antiques de Vénus, déesse italique issue de l'Antiquité païnne et retravaillée au Moyen Âge, à l'instar d'autres divinités telles Amour, Fortune et Mort. Derrière le nom de Vénus existe toute la richesse d'un passé culturel gréco-romain, que Le Livre du Cuer d'Amours espris suggère en filigrane, contrairement au Roman de la Rose qui inclut des rappels mythologiques précis de son histoire dans le récit ${ }^{4}$.

Divinité prestigieuse et puissante, elle était respectée et faisait l'objet de grands cultes. Déesse de l'amour et de la beauté assimilée à la grecque Aphrodite, elle est la Vénus aux mille charmes narrés par les Anciens, d'Homère à Ovide en passant par Lucrèce et Virgile. L'amour, la beauté, cette double dimension est reprise par René d'Anjou qui imagine un château si beau qu'il semble davantage « chose celestielle ou espirituelle que terrienne " cristallisation de l'amour du dieu envers elle :

«C'est le beau chastel de Plaisance ;

La fait Amours demourance.

Faire le fist plus bel que nulz

Pour l'amour de dame Venus. $»^{6}$

$5 \quad$ Fille de Jupiter et de Dioné selon les légendes, ou née de l'écume des flots marins ainsi que le représente Sandro Botticelli dans son tableau intitulé « La Naissance de Vénus » (1485), après la castration d'Uranus par son fils Saturne ${ }^{7}$, elle est l'épouse de Vulcain, le dieu du feu. Comme l'indique le substantif "venus, veneris» qui signifie "charme, attrait, élégance, grâce ", Vénus exerce un grand pouvoir de séduction sur les hommes, qu'ils soient dieux ou mortels, art qu'elle tient des Heures qui l'ont accueillie et éduquée sur l'île de Chypre.

6 Le roi de Sicile évoque en outre la filiation de la déesse avec Enée, fruit de ses amours avec le troyen Anchise. La devise de l'écu qu'Enée a déposé au portail du cimetière de l'hôpital d'Amour commence ainsi :

« Henee troyen suys, filz d'Anchises mon pere, Comme l'ouÿ conter a Venere ma mere. $\|^{8}$

7 L'emploi du terme latin « Venere » accentue la coloration antique de la figure de Vénus, autorité qui garantit l'origine divine des fils d'Enée, les Romains.

8 Mais l' " aeneadum genetrix » invoquée par Lucrèce est également la mère de Cupidon, dieu de l'amour aussi appelé Amour et identifié au grec Eros. Cette dernière généalogie est particulièrement exploitée au Moyen Âge, notamment par René d'Anjou.

\section{Une autorité à la cour d'Amour}

9 L'occupation du château d'Amour par Vénus témoigne de son autorité dans le domaine de l'amour. Nous tenterons de montrer que son champ d'action demeure étroitement lié à son espace relationnel. 
10 Dans Le Livre du Cuer d'Amours espris, Vénus est avant tout désignée par rapport à son fils : « sa tredoulce mere Venus $»^{9}$, « Venus sa mere ${ }^{10}$, « son filz le dieu d'Amours ${ }^{11}$, « dame Venus sa mere ${ }^{12}$. Il apparaît, au regard de ces citations, que la filiation est l'élément qui lui confère de l'autorité : elle est la mère du dieu Amour. Elle incarne une autorité maternelle qui inspire respect et crainte, attitude que lui témoigne son fils : « son filz le dieu d'Amours, qui lui fist grant reverence $»^{13}$. Mais cette relation auctoriale mère/fils devient universelle, elle est la mère de tous, comme le montrent le pronom possessif pluriel « nostre » et le verbe « devoir» :

«A nostre mere yrons parler,

Venus, que devons bien amer. $»^{14}$

11 Dans le récit de cette quête amoureuse inspirée du modèle du Roman de la Rose de Guillaume de Lorris et Jean de Meun, dame Vénus occupe une place justifiée aux côtés d'Amour. Le couple mère/fils - Vénus/Amour impose son autorité comme représentant du pouvoir de l'amour : alors que Bel Accueil, emprisonné par la naine Jalousie, va être libéré par dame Espérance, il invoque les deux puissances :

« (...) elle ouÿt la voix d'une personne qui fort se plaignoit a voix basse et cassee et reclamoit fort le dieu d'Amours et sa tresdoulce mere Venus. ${ }^{15}$

Dame Pitié également ne dissocie pas les deux figures :

«Si lui conseilla le Cuer en l'oreille que il l'en vouloit mener au chastel de Plaisance, et la useroient leurs jours en la compaignie d'Amours et de dame Venus sa mere. $»^{16}$

Amour et Vénus s'esquissent comme deux forces qui régissent l'univers amoureux :

«(...) vint ung jouvencel qui dist a Amours que dame Pitié, la prieuse de l'ospital d'Amours, estoit venue leans. Et Amours respondit que bien fust elle venue, et commanda qu'elle fust menee a Venus sa mere, et ceulx a qui il fut commandé le firent incontinent. » 17

Mais l'île, le château de Plaisance, la cour, le conseil appartiennent à Amour, ou du moins, sont associés à son nom. L'autorité d'Amour est mise en lumière, tandis que Vénus se tient dans l'ombre de son fils. Sa présence reste en effet discrète et aucune de ses paroles n'est retranscrite. Cependant, elle inspire obéissance et jouit d'une grande autorité sur son enfant et sur les autres figures. La citation suivante illustre ce paradoxe :

« Il ne demoura gueres que dame Venus entra en la chambre, car elle avoit a coustume de venir veoir tousjours aprés disner et soupper son filz le dieu d'Amours, qui lui fist grant reverence. Et a chief de piece le tira a part et appela dame Pitié, qui avec elle estoit venue en la chambre. Puis appela Loyauté, Honneur, Bon Renom, Vaillance, Bel Acueil et pluseurs autres de son conseil et fist vuidier tout homme. $»^{18}$

15 On remarque d'ailleurs que l'attitude du chevalier Cœur est différente à l'égard de Vénus : il témoigne de son respect et de son obéissance envers celle-ci, alors que rien de tel ne nous est dit par rapport à Amour :

« (...) le Cueur mist le genoil a terre aprés ce qu'il eut salué et fait reverence a dame Venus, et dist a Amours ainsi (...). $»^{19}$

16 Ancienne déesse de l'amour et mère savante ${ }^{20}$, elle est une pièce maîtresse sur l'échiquier amoureux. Elle n'intervient pas physiquement dans la psychomachie amoureuse ${ }^{21}$, mais sa parole fait autorité. C'est pourquoi elle siège au conseil d'Amour :

« Mais dame Oyseuse leur respondit que le dieu d'Amours, son sires, estoit pour celle heure en bien estroit conseil, ouquel n'y avoit nulz que sa mere premier et deux des plus 
espiciaulx et secretz serviteurs de ceulx de son conseil avecques ung secretaire, ainsi pour voir qu'elle avoit ouÿ dire en la chambre de dame Venus, de laquelle ne faisoit que venir, cuidant baignier son faulcon en ladicte fontaine. ${ }^{22}$

17 La présence indispensable de sa mère - « sa mere premier » - dans cet « estroit conseil » montre qu'elle occupe une place privilégiée dans la politique amoureuse menée par son fils. En exerçant une influence sur Amour, Vénus influe sur les affaires d'amour.

Le dieu ne peut prendre de décision sans se référer à cette autorité, dont l'opinion et le conseil sont prioritaires :

« Nous y aurons ung peu d'advis

$O$ noustre conseil et amis.

A nostre mere yrons parler,

Venus, que devons bien amer. ${ }^{23}$

«Cy vous a devisé le conte l'estre de ladicte salle et pour tant s'en taist et revient a parler d'Amours, lequel est ou conseil, qui demanda l'advis, premier a dame Venus sa mere, et puis a tous les autres par ordre, de ce que le Cueur lui avoit fait proposer et requerir par Desir, comme vous avez ouÿ. $\|^{24}$

Le geste du toucher de la main qui « sert à confirmer un pacte ou un contrat ${ }^{25}$, souligne encore le poids de dame Vénus dans la politique de la cour d'Amour :

« Il fut temps de soupper et dame Venus se voulloit retraire en sa chambre. Mais premier prirent congié d'elle les compaignons de la queste de Doulce Mercy, car ilz se vouloient partir bien matin pour achever ce qu'ilz avoient a faire, et elle leur toucha a chascun en la main et les commanda a Dieu benignement. $»^{26}$

\section{L'autorité des tapisseries de Vénus}

20 L'épisode des huit tapisseries de Vénus approfondit notre enquête sur l'autorité de cette figure et son enjeu narratif. La déesse cautionne les idées exposées par les tapisseries, elle certifie et prouve l'authenticité et la véracité des scènes représentées et des vers qui les accompagnent, car elles les a disposées dans sa chambre. En outre, elle autorise indirectement l'Acteur à parler de ses tapisseries à travers une analogie avec ses vêtements, dont la richesse et la magnificence rappellent ceux qui lui ont été donnés par les Heures :

«Si commença a marcher droit a sa chambre si gentement que bel la faisoit veoir. De ses habillemens ne vous vieulx je parler pour cause de briefveté ; et aussi mon engin n'est pas suffisant de les savoir diviser, mais tant vous di je bien qu'elle estoit belle et habillee comme une deesse. La tapisserie de sa chambre estoit toute de satin cramoisy, brodé de fin or et de perles, a personnages telz que cy aprés s'ensuivent. $»^{27}$

21 Le souci de brièveté et le topos de l'indescriptible servent de prétexte poétique. René d'Anjou substitue au topos descriptif de la tenue de la dame un motif décoratif de sa chambre : l'énumération des tapisseries ${ }^{28}$. Leurs inscriptions sont composées de cinq vers, nombre que nous pourrions interpréter comme la marque personnelle de Vénus, dont la première lettre du nom représente le chiffre romain cinq.

Plus encore, les tapisseries se placent sous l'autorité d'un épisode mythologique tiré de l'histoire de Vénus : ses amours avec Mars, le dieu de la guerre. Sur le modèle des 
Métamorphoses d'Ovide ${ }^{29}$, Le Roman de la Rose nous conte comment Vénus est capturée pendant un acte d'adultère avec Mars par un filet invisible tressé par son mari Vulcain :

« Es laz qu'il ot d'arain forgiez

Les tenoit andeus enfergiez,

El gieu d'amours joins et liez,

Tant les ot li fols espiez.

Si tost com Vulcanus le sot,

Que pris prouvez andeus les ot

Es laz qu'entour le lit posa

Mout fu fols quant faire l'osa,

Car cil a mout pou de savoir

Qui seuls cuide sa fame avoir-

Les dieus i fist venir en heste,

Qui mout ristrent et firent feste

Quant en tel point les aperçurent. ${ }^{30}$

La liaison entre Vénus et Mars va colorer l'orientation thématique des tapisseries, qui se lisent comme des représentations de la capture amoureuse, motif traditionnel au Moyen Âge ${ }^{31}$. La capture des amants mythiques sert ainsi d'exemple pour devenir à la fois un motif esthétique et didactique : René d'Anjou convoque l'autorité de Vénus pour tisser une tapisserie édifiante, dirigée par deux images. La première est celle du filet d'amour ${ }^{32}$, véritable piège multiforme : « le bray du souvenir $»^{33}$, « la reiz saillant (...) et tendu $»^{34}$, les « lassetz d'acointance (...) tendus es pas $»^{35}$, « la pentiere » tendue « au coing du boys $»^{36}$, les «cages » de «soucÿes » et d' " ancolies ${ }^{37}$ construites de fils d'acier verticaux et horizontaux tels le filet de cuivre fabriqué par Vulcain. Dans la cinquième tapisserie, le piège utilisé n'est pas fait à base de fil mais de glue : Folle Présomption et Espérance « Ont englué ung hault arbre d'abus $»^{38}$, stratagème que l'on trouve dans la Farce de la Pipée $e^{39}$.

La seconde image est celle du cœur ailé. Elle évoque les enluminures du Livre du Cuer d'Amours espris qui identifient le chevalier Cœur à son heaume garni d'un cœur rouge ailé, ce qui transforme la lecture de ces tapisseries en une série de mises en abîme du Cœur lui-même. Elle fait d'autre part allusion à Hermès, célèbre amant de Vénus aux sandales et au casque ailés ou encore à Amour, son fils pourvu d'ailes, comme le représente l'enluminure du fol. 2 du ms. de Vienne ${ }^{40}$. "A happer cueurs vollages ${ }^{41}$, cette métaphore courante au Moyen Âge désigne le vol des cœurs ailés, mais aussi les cœurs volages, infidèles, sur le modèle de Mars et Vénus. On retrouve cette même idée dans la quatrième tapisserie à travers l'expression « cueur volant peu estable $»^{42}$ qui évoque les cœurs peu fidèles.

Une comparaison entre les enluminures tirées du ms. fr. 24399, fol. 122-123, du Livre du Cuer d'Amours espris qui dépeignent la façon dont les cœurs, représentés de façon stylisée, se jettent dans les pièges disposés, comme attirés par un magnétisme pareil à celui de Vénus, et celles du ms. Morgan 948, fol. 135r, et du ms. Douce 195, fol 99r, du Roman de la Rose de Guillaume de Lorris et Jean de Meun, qui représentent Vénus et Mars enlacés et pris au piège de Vulcain, renforcent l'autorité de cet épisode. Possédant la connaissance et l'expérience de l'amour, Vénus est une référence convoquée pour traiter de la séduction et des pièges de l'amour.

Enfin, dans les septième et huitième tapisseries, apparaissent deux autres figures de l'autorité : la personnification épicurienne Roger Bon Temps, qui a préféré se retirer du 
service d'Amour, et le sage Vieillard, dont la parole autoritaire résonne depuis des temps anciens et à travers tout le Moyen Âge.

\section{NOTES}

1. LUCRÈCE, De Natura Rerum, texte établi, introduit et annoté par A. ERNOUT, Paris, Les Belles Lettres, 1962, Livre 1, vers 1-5: "Mère des Enéades, plaisir des hommes et des dieux,Vénus nourricière, toi qui, sous les signes errants du ciel, peuples la mer porteuse de vaisseaux, les terres riches en moissons, puisque par toi toute l'espèce des êtres animés est conçue et vient voir, une fois née, les rayons du soleil ».

2. RENÉ D'ANJOU, Le livre du coeur d'amour épris, texte présenté, établi, traduit et annoté par F. BOUCHET, Paris, Librairie Générale Française, 2003, vers 2181.

3. Ibid., p. 452.

4. Vénus et Adonis, Pygmalion, l'adultère de Vénus et la castration de Saturne.

5. RENÉ D'ANJOU, op .cit., p. 394.

6. Ibid., vers 1135-1138.

7. Dans Le Roman de la Rose de Guillaume DE LORRIS et Jean DE MEUN, texte édité et traduit par Armand strubel, Paris, Garnier Flammarion, 1992, vers 5531-5537, c'est l'émasculation de Saturne par Jupiter qui provoque la naissance de Vénus : «Joustice qui jadis regnot/Au tans que Saturnus regne ot,/Cui Jupiter coupa les coilles,/Ausi com se fussent andoilles/-Mout ot cil dur fill et amer-/Puis les gita dedenz la mer/Dont Venus la deesse issi ».

8. RENÉ D'ANJOU, op. cit., vers 1279-1280.

9. Ibid., p. 146.

10. Ibid., p. 434.

11. Ibid., p. 434-436.

12. Ibid., p. 444.

13. Ibid., p. 434-436. L'autorité maternelle de Vénus est explicitée dans Le Roman de la Rose, op.cit., vers 10761-10764: " Ma mere est, si la creing d'enfance;/Je li port mout grant reverance,/ K'enfezs qui ne craint pere et mere/Ne puet estre qu'il nel compere ».

14. RENÉ D'ANJOU, op. cit., vers 2097-2098.

15. Ibid., p. 146.

16. Ibid., p. 488.

17. Ibid., p. 434.

18. Ibid., p. 434-436.

19. Ibid., p. 446.

20. Le Roman de la Rose, op. cit., vers 10747-10748, rejoint cette dimension : « Vostre mere qui mout est sage,/Qu'ele set trop de cest usage ».

21. Dans Le Roman de la Rose, op. cit., Vénus intervient notamment pour aider les troupes de l'armée d'Amour. Elle met le feu à la forteresse et permet la victoire finale de l'Amant.

22. RENÉ D'ANJOU, op. cit., p. 420-422.

23. Ibid., vers 2095-2098.

24. Ibid., p. 444.

25. Florence BOUCHET, Le livre du cœur d'amour épris, op. cit., note 1, p. 453. Voir A. J. GREIMAS et T.

M. KEANE, Dictionnaire du moyen français, Paris, Larousse, 1992, p. 628. 
26. RENÉ D'ANJOU, op. cit., p. 452.

27. Ibid., p. 452.

28. Cette transposition d'intérêt poétique correspond à l'engouement du $\mathrm{XV}^{\mathrm{e}}$ siècle pour les tapisseries et les tentures.

29. ovIDE, Les Métamorphoses, texte établi et traduit par G. LAFAYE, éd. G. LAFAYE et H. LE BONNIEC, Paris, Les Belles Lettres, 2002, Livre 4, vers 167-189.

30. GUILLAUME DE LORRIS et JEAN DE MEUN, Le Roman de la Rose, op. cit., vers 13847-13859.

31. Dans son Traité de l'amour courtois, texte introduit, traduit et annoté par C. BURIDANT, Paris, Klincksieck, 1974, p. 49, ANDRÉ LE CHAPELAIN fournit l'analyse étymologique suivante du terme « amour » : « Amour vient du verbe aimer qui signifie « prendre » ou « être pris ». Car celui qui aime est pris dans les chaînes du désir et il souhaite prendre l'autre à son hameçon. De même en effet que le pêcheur adroit s'efforce de ferrer les poissons grâce à ses appâts, et de les prendre à son hameçon crochu, de même celui qui est pris par l'amour s'ingénie à attirer l'autre par des manœuvres de séduction et il s'applique de toutes ses forces à unir deux cœurs différents par un lien impalpable ou à les maintenir unis pour toujours s'ils le sont déjà ".

32. On trouve aussi cette image dans Le Roman de la Rose, op. cit., vers 1437-1438: « Narcisus fu .i. damoisiaus/Qu'amors mist en ses raiseaus ».

33. RENÉ D'ANJOU, op. cit., vers 2260.

34. Ibid., vers 2265-2266. Nous noterons toutefois que la miniature de cette tapisserie montre plutôt l'image bucolique de deux jeunes femmes attrapant les cœurs comme elles attraperaient des papillons à l'épuisette.

35. Ibid., vers 2270 .

36. Ibid., vers 2276 .

37. Ibid., vers 2284-2285.

38. Ibid., vers 2280 .

39. M. ROUSSE, «L'allégorie dans la farce de la Pipée », Cahiers de l'Association internationale d'études française, Paris, Association internationale des études françaises, 28, 1976, p. 37-50. Les oiseaux identifiés comme les galants sont pris au piège inventé par Plaisant Follie et Cuider. Citons aussi CHARLES D'ORLÉANS, Ballades et Rondeaux, éd. Jean-Claude MÜHLETHALER, Paris, Librairie Générale Française, 1992, Rondeau 69, vers 8-10 : «Fortune par sez faulz atrais/En pipant a pris a la glus/ Mon cueur (...)».

40. Vienne, National-bibliothek, cod. Vindobonensis 2597.

41. RENÉ D'ANJOU, op. cit., vers 2267.

42. Ibid., vers 2278 .

\section{INDEX}

Mots-clés : autorité, amour, paganisme, déesse, Antiquité, Vénus, Anjou

Keywords : authority, love, paganism, goddess, Antiquity, Venus, Anjou 